\title{
Mean field and Monte Carlo simulation studies of premartensitic effects in $\mathrm{Ni}_{2} \mathrm{MnGa}$
}

\author{
T. Castán, E. Vives and P.-A. Lindgård ${ }^{1}$ \\ Departament d'Estructura i Constituents de la Matèria, Facultat de Física, \\ Universitat de Barcelona, Diagonal 645, 08028 Barcelona, Catalonia, Spain \\ ${ }^{1}$ Condensed Matter Physics and Chemistry Department, Risø National Laboratory, \\ 4000 Roskilde, Denmark
}

\begin{abstract}
We have extended the degenerate BEG model[1] to include magnetic degrees of freedom in order to study the premartensitic effects in $\mathrm{Ni}_{2} \mathrm{MnGa}$. The model is solved by using mean-field theory and Monte Carlo techniques [2]. The numerical simulations reveal the crucial importance of fluctuations in pretransitional effects. Moreover, we find that a large variety of premartensitic effects may appear due to the magnetoelastic coupling. For large values of the coupling parameter a first-order transition line ending in a critical point appears. This critical point is responsible for the existence of large premartensitic fluctuations which manifest as broad peaks in the specific heat, not always associated with a true phase transition. The main conclusion is that premartensitic effects result from the interplay between the softness of the anomalous phonon driving the modulation and the magnetoelastic coupling strength. In particular, the premartensitic transition occurs when such coupling is strong enough to prevent a complete softening of the involved phonon mode. The implication of the results in relation to the available experimental data is discussed.
\end{abstract}

\section{INTRODUCTION}

Many metals and alloys undergo a so called martensitic transition (MT) from an open cubic phase at high temperatures to a more closed packed phase at lower temperatures [3]. It is a displacive, diffusionless, first-order phase transition, accompanied by incomplete softening of certain transverse phonon modes. It has been suggested that the first-order character can be understood as an effect of a coupling between two simultaneous strains: an internal two-plane shuffle strain $(\eta)$ and a uniform $\operatorname{strain}(\epsilon)[4]$.

A rich variety of precursor phenomena [5] have been observed in (weakly) first-order MT. The most significant is the anomalously low $T A_{2}[110]$ phonon branch ([110] propagation, [110] polarization]), accompanied by a low value of the elastic constant $C^{\prime}=\left(C_{11}-C_{12}\right) / 2$. Moreover, both the phonon branch and the corresponding elastic stiffness soften with temperature. Recently, a lot of interest has been focused on the intermetallic Ni-Mn-Ga alloys close to the stoichiometric composition $\mathrm{Ni}_{2} \mathrm{MnGa}$. It is the only known ferromagnetic fcc $\left(\mathrm{L} 2_{1}\right)$ Heusler alloy undergoing a MT at a temperature $T_{M}$ below the Curie temperature $T_{m}$. Besides its theoretical interest, it may be of technological importance too since it opens the possibility of controlling its shape memory properties (intimately related to the MT) by applying an external magnetic field [6]. In particular the quest for finding systems with very large magnetostrains is one of the objectives in order to develop a new class of actuator materials. At the MT the structure of the system changes from a cubic phase (which can be viewed as a bcc when neglecting the atomic order) to a low temperature tetragonal phase bct, which is modulated by a five-plane shuffle strain $[7,8]$. Particularly intriguing is that for nearly stoichiometric composition the full MT may be preceded by an intermediate phase transition (IT) at $T_{I}$ in which 
apparently only the shuffle strain is activated, but not the tetragonal strain(the cubic symmetry is preserved) $[9,10,11,12]$. This is accompanied by a significant, although not complete, softening of the $T A_{2}$ phonon branch at a wave vector $\xi_{0}=0.33$. Only at lower temperatures, at the martensitic transition point $T_{M}$, the homogeneous tetragonal strain is activated (and the modulation changes slightly). This particular behavior observed in $\mathrm{Ni}_{2} \mathrm{MnGa}$ seems to be related to the influence of magnetism. The point is whether or not this intermediate modulated structure is a genuine new phase. Different behaviors have been reported in the literature. For some samples $[9,11,12]$ there exists evidence for a true phase transition at $T_{I}$ of very weak first-order which is driven by a magnetoelastic coupling. The main proof of that is the fact that $T_{I}$ shifts with the external applied field [9] while no (significant) magnetic field dependence has been found for the $T_{M}$ temperature $[13,15]$. Moreover, the magnetoelastic effects have been confirmed from the experimentally observed dependence of the elastic constants on an external magnetic field [16]. In other studies [14], the authors could not find any indication for an intermediate phase although precursors, clearly related to the magnetization of the sample, have been observed. The temperatures $T_{m}, T_{M}$ and $T_{I}$ are extremely sensitive to the

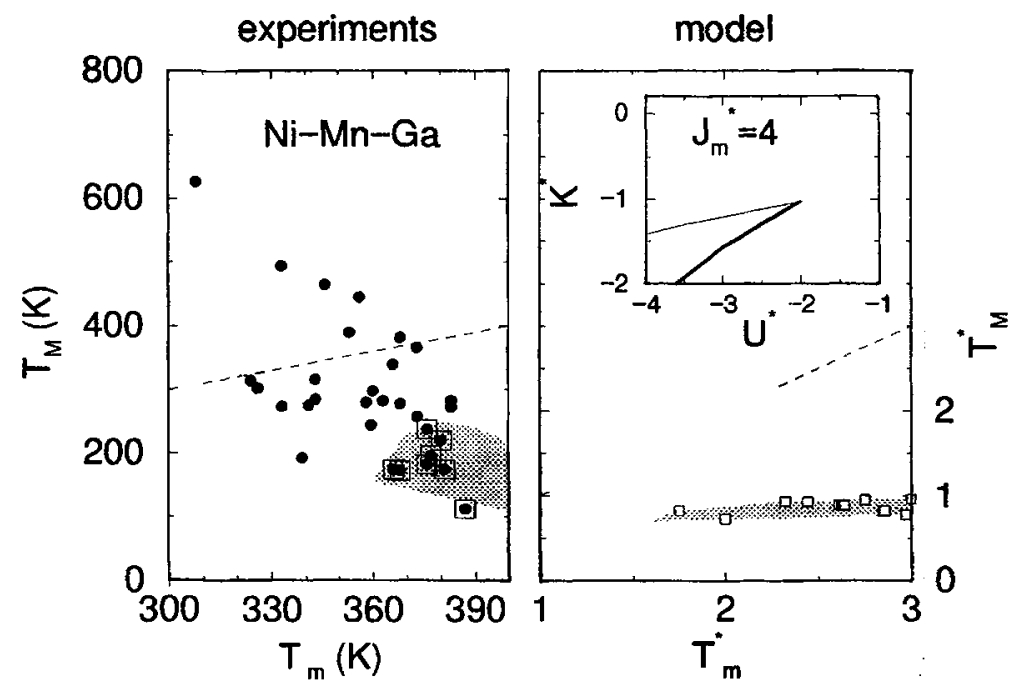

Figure 1: (a) Curie temperatures $T_{m}$ and martensitic transition temperatures $T_{M}$ for different samples in the bibliography. Squares indicate those samples which exhibit an intermediate premartensitic phase transition and the dashed area the zone where this occurs. The dashed line indicates the condition $T_{M}=T_{m}$. (b) Same graph obtained from the present model by scanning the three model parameters $U^{*}, J_{m}^{*}$ and $K^{*}$. The dashed area indicates approximately the region where the existence of a premartensitic transitions is predicted by MF. The white squares indicates the actual numerical data obtained. The inset shows the same region in the model parameters space (for $\left.J_{m}=4\right)$. The thick line indicates the premartensitic critical points.

composition and atomic ordering of the sample. The Curie point $T_{m}$ may vary between $360 \mathrm{~K}$ to $395 \mathrm{~K}$, whereas $T_{M}$ may vary from $175 \mathrm{~K}$ to $450 \mathrm{~K}$. For instance in the sample studied by Stuhr et al. [14], $T_{m} \approx 364 \mathrm{~K}$ and $T_{M} \approx 284 \mathrm{~K}$ with precursor signs for $T>T_{M}$, but no intermediate phase was found. In the sample studied by Zheludev et al. [11, 12] $T_{m} \approx 380 \mathrm{~K}$ and $T_{M} \approx 220 \mathrm{~K}$ and an intermediate phase is observed below $T_{I} \approx 260 \mathrm{~K}$. Similarly, Planes et al [9] studied a sample with $T_{m} \approx 381 \mathrm{~K}$ and $T_{M} \approx 175 \mathrm{~K}$ and found an intermediate transition at $T_{I} \approx 230 \mathrm{~K}$. Apparently, the 
only relevant difference in the samples used by Planes et al [9], Zheludev et al [11, 12] and Stuhr et al [14] is the content in $\mathrm{Mn}$. Figure 1(a) represents the values of $T_{M}$ and $T_{m}$ corresponding to different samples reported in the literature. The dots surrounded by squares correspond to samples so far found exhibiting the intermediate transition IT. They appear to fall in a particular zone in the $T_{M}-T_{m}$ diagram.

In this paper we present a microscopic theoretical model aimed to investigate the role of the magnetic coupling in $\mathrm{Ni}-\mathrm{Mn}-\mathrm{Ga}$. The model has been solved by means of both Mean-Field (MF) and Monte Carlo (MC) techniques and we have obtained that both methods render the same qualitative agreement. The MC simulations allow an analysis of fluctuations, beyond previous mean-field (MF) or Landau expansion treatments $[6,9,18]$. The model is an extension of the degenerate BlumeEmery-Griffiths model (DBEG) [1] which includes a coupling to magnetic degrees of freedom.

\section{THE MODEL}

The desired degrees of freedom can be represented by the $p$-degenerate Blume-Emery-Griffiths Hamiltonian (DBEG)[1]. It was first introduced with the aim to account for the entropy stabilization of the high temperature phase in martensitic transitions. It is an extension of the ordinary three-state Blume-Emery-Griffiths Hamiltonian defined on a lattice, which we shall take as simple cubic (or square). On each lattice site $i=1, \ldots, N$ a variable $\sigma_{i}=1,0,-1$ represents the deformation state near each site on the lattice. The state $\sigma_{i}=0$ represents the undistorted phase, and it is chosen to be $p$-fold degenerate $(p \geq 1)$, in order to approximately account for the high entropy of vibration of the cubic phase. The states $\sigma_{i}= \pm 1$ represent the distorted phase. The Hamiltonian accounting for the energy gain in having the same structure on neighboring sites was written as [1]

$$
\mathcal{H}_{M}=-J \sum_{\langle i, j\rangle}^{n . n .} \sigma_{i} \sigma_{j}-K \sum_{\langle i, j\rangle}^{n . n .}\left(1-\sigma_{i}^{2}\right)\left(1-\sigma_{j}^{2}\right),
$$

where the sums are performed over all nearest-neighbor pairs. In what follows we will take $J>0$ as the unit of energy and work in terms of reduced magnitudes defined as $\mathcal{H}_{M}^{*}=\mathcal{H}_{M} / J, K^{*}=K / J$, $T^{*}=k_{B} T / J, \ldots$ For $\mathrm{Ni}_{2} \mathrm{MnGa}$ is natural to define the following two order parameters:

$$
\epsilon=\frac{\sum \sigma_{i}}{N} \quad q=\frac{\sum \sigma_{i}^{2}}{N} .
$$

The symmetry breaking order parameter $\epsilon$ corresponds to the tetragonal distorsion in the sense that if $\epsilon=0$ (equal population of $\sigma_{i}=+1$ and -1 ) it corresponds to having all the variants equally populated, and hence an average cubic phase. For $q$ the relation is more involved because of the complicated physics of the modulating strain $\tilde{\eta}$. In the high temperature cubic phase, when the $\sigma_{i}$ variables distribute at random $q=q_{0}=2 /(p+2)$. Let us assign the difference $q_{0}-q$ with the amplitude $\tilde{\eta}$ of the plane modulating strain.

We now include the magnetic degrees of freedom by means of spin variables $S_{i}= \pm 1$ (defined on the lattice site $i=1, . ., N$ ) having a ferromagnetic Ising interaction. Thus, the purely magnetic contribution is:

$$
\mathcal{H}_{m}^{*}=-J_{m}^{*} \sum_{\langle i, j\rangle} S_{i} S_{j},
$$

where $J_{m}^{*}>0$. The total Hamiltonian should further include a coupling term between the structural and magnetic variables. We assume that the magnetic influence of electronic properties gives rise to a coupling between the magnetic moment and the plane modulation. To incorporate this into the 
description, the interaction contribution that should be considered is:

$$
\mathcal{H}_{\mathrm{int}}^{*}=U^{*} \sum_{\langle i, j\rangle} S_{i} S_{j}\left[\sigma_{i}^{2}\left(1-\sigma_{j}^{2}\right)+\sigma_{j}^{2}\left(1-\sigma_{i}^{2}\right)\right]
$$

with $U^{*}<0$. In mean-field approximation, this term becomes of the form $m^{2} \eta^{2}$ in a Landau expansion plus a simple modification of the purely magnetic interaction. The total Hamiltonian model can then be written as

$$
\mathcal{H}^{*}=\mathcal{H}_{M}^{*}+\mathcal{H}_{m}^{*}+\mathcal{H}_{\text {int }}^{*}
$$

We shall demonstrate that it is possible to split up the structural transition into one determined by the order parameter $q$, which we will associate with the IT and another one, determined by $\epsilon$, to be associated with the tetragonal deformation occurring at the MT.

\section{RESULTS}
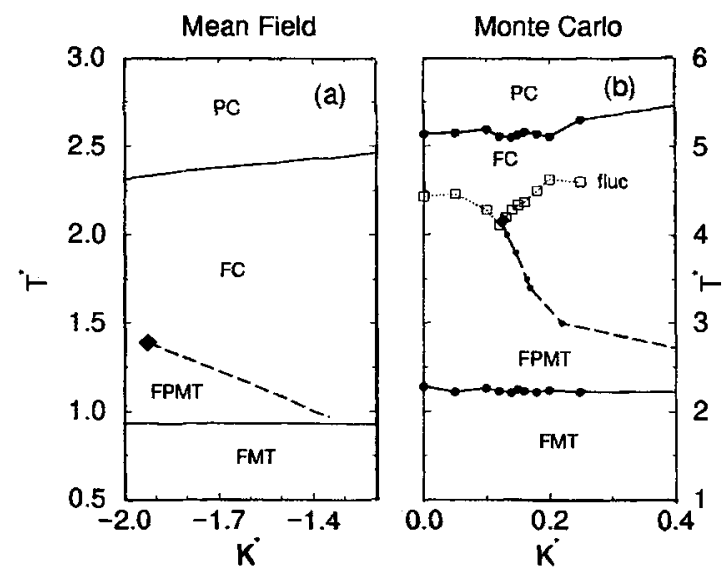

Figure 2: Phase diagrams corresponding to $J_{m}^{*}=4$ and $U^{*}=-3.5$. (a) Mean field (b) Monte Carlo. In both cases the dashed line stands for first order premartensitic phase transitions, the black diamond indicates the critical point and the empty squares indicate large critical fluctuations.

A detailed description of the MF and MC solution of the model in Eq. (5) has been published recently [2]. Here we focus on the analysis of the main properties of the obtained phase diagrams for $p=4$. An example of a $T^{*}-K^{*}$ section is presented in Figure 2. The same features are observed in both meanfield theory and Monte Carlo simulations. The value of the ferromagnetic interaction parameter $J_{m}^{*}=4>0$ determines the distance between the magnetic $T_{m}^{*}$ and the martensitic $T_{M}^{*}\left(<T_{m}^{*}\right)$ transitions. For appropriate values of the parameters $K^{*}$ and $U^{*}$, the following phases are found, from high to low temperature: paramagnetic cubic (PC), ferromagnetic cubic (FC), ferromagnetic intermediate or premartensitic (FPMT) and ferromagnetic martensitic (FMT). The change from the FC to the FPMT phases occurs below a critical point $\left(T_{I c}^{*}, K_{c}^{*}\right)$ and it takes place trough a true phase transition only for $K^{*}>K_{c}^{*}$. The existence of this intermediate phase depends on both $K^{*}$ and $U^{*}$ (see inset in Fig. 1(b)). First one needs the magnetoelastic coupling $U^{*}\left(-J_{m}^{*}<U^{*}<0\right)$ to be strong enough. We obtain that $T_{I c}^{*}$ decreases with $U^{*}$ whereas $T_{M}^{*}$ remains almost unaltered so that the critical point disappears, on the $T_{M}^{*}$ line, well before $U^{*}$ reaches the value zero. Next, 
provided the $U^{*}$ is adequate, the IT exists for a limited range of values of $K^{*}\left(>K_{c}^{*}\right)$ across the first-oder transition line $T_{I}^{*}\left(K^{*}\right)$. This is illustrated in the inset of Fig 1(b) for a particular value of the magnetic interaction $J_{m}^{*}=4$.

In Fig. 3(a), we show the MF temperature behaviour of the order parameters below (a), above (c) and at the critical $K_{c}^{*}=-1.93$ (b). The modulating strain amplitude $\eta=q_{0}-q$ changes from high to low values as one decreases the temperature across the IT. According to the theory of the harmonic thermal vibrations in a crystal, this is consistent with the behavior observed for the phonon frequency [12]. Moreover, in our results, the IT is accompanied by a jump in the magnetization. Since this happens in both mean-field and Monte Carlo solutions we conclude that this has to do with the coupling rather than with the fluctuations. Experimentally, a jump in the magnetization has not been detected so far $[17,18]$.

Monte Carlo simulations reveal the important role played by the fluctuations in describing premartensitic effects. Fig. 3(b) shows the temperature behaviour of the specific heat for different values of $K^{*}$ and below the $K_{c}^{*} \simeq 0.13$. The peaks obtained at different intermediate temperatures between $T_{M}^{*}$ and $T_{m}^{*}$ (indicated by arrows) are associated with premartensitic effects. The narrow peak (on the left) corresponds to the true IT, whereas the wide one (on the right) corresponds to critical fluctuations. This last peak is only visible in the vicinity of the critical point and is not associated with a true phase transition. The position of such peaks has been indicated on the phase diagram in Figure 2(b) by empty squares.
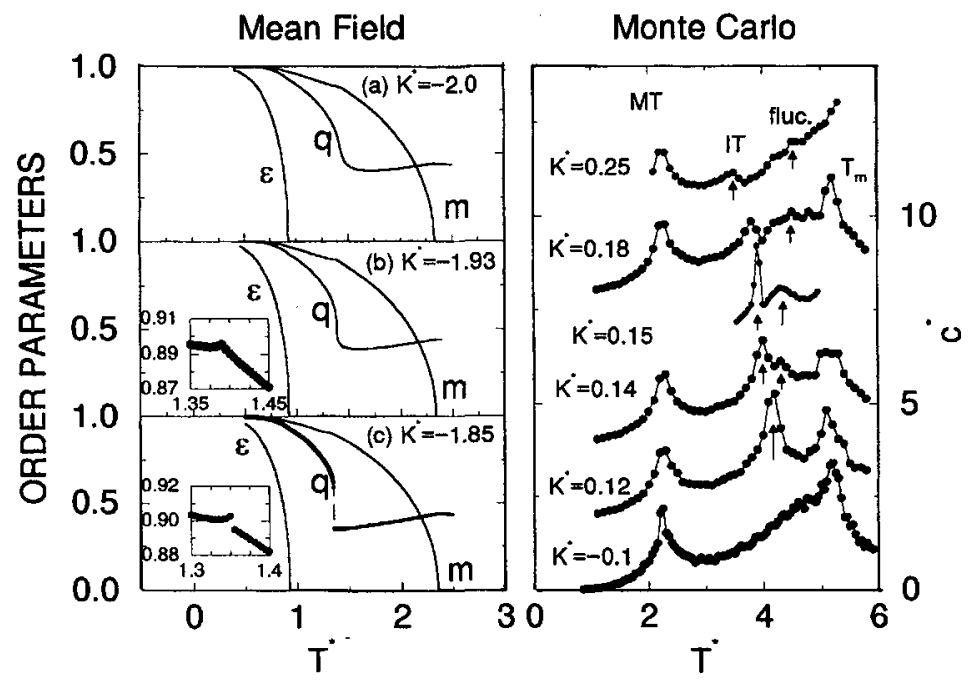

Figure 3: Order parameters calculated using MF theory, (the insets show a magnification of $m$ ), and the specific heat obtained by MC simulations for $J_{m}^{*}=4.0$ and $U^{*}=-3.5$ and different values of $K^{*}$ as indicated.

\section{CONCLUSIONS}

In summary, the model reproduces the existence of different premartensitic effects controlled by the interplay between the magnetoelastic coupling $U^{*}$ and the strength of the phonon interaction $K^{*}$. The region of model parameters for which a premartensitic transition appears in MF theory can 
be translated into a $T_{M}^{*}-T_{m}^{*}$ diagram which is shown in figure $1 \mathrm{~b}$ by a dashed zone. Note that experimentally (Fig. 1(a)) this region is limited to high values of $T_{m}$ as well.

The model studied by MC simulations predicts that the magnetoelastic coupling may give rise to premartenstic effects associated with large critical fluctuations which should not be attributed to a genuine phase transition. Experimentally, the interplay between $U^{*}$ and $K^{*}$, required for the IT to occur, is determined by the composition of the sample. Indeed, premartensitic effects in the $\mathrm{NiMnGa}$ alloy have only been reported for compositions around the stoichiometric $\mathrm{Ni}_{2} \mathrm{MnGa}$. The intermediate structure may appear through a true phase transition $[9,12]$ or not [14]. We point out that both behaviors are compatible with the present results.

\section{Acknowledgments}

This work has received financial support from CICyT (project number MAT98-0315).

\section{References}

[1] E. Vives, T.Castán and P.-A.Lindgård, Phys. Rev. B 53, 8915 (1996).

[2] T.Castán, E.Vives and P.-A.Lindgård, Phys. Rev. B 60, 7071 (1999).

[3] Z. Nishiyama, Martensitic Transformations Academic Press, New York, 1978).

[4] P. -A. Lindgård and O. G. Mouritsen, Phys. Rev. Lett. 57, 2458 (1986).

[5] J. A. Krumhansl and Y. Yamada, Mat. Sci. Engng. A127, 167 (1990).

[6] A.N. Vasil'ev, A.D. Bozhko, V.V. Khovailo, I.E. Dikshyein, V.G. Shavrov, V.D. Buchelnokov, M. Matsumoto, Phys. Rev. B59, 1113 (1999).

[7] V.V.Martynov, V.V.Kokorin, J. Phys. III (France) 2, 739 (1992).

[8] G.Fritsch, V.V.Kokorin and A.Kempf, J.Phys. : Condens. Matter 6, L107 (1994).

[9] A.Planes, E.Obradó, A.Gonzalez-Comas and Ll. Mañosa, Phys. Rev. Lett. 79, 3926 (1997).

[10] Ll.Mañosa, A.Gonzàlez-Comas, E.Obradó, A.Planes, V.A.Chernenko, V.V.Kokorin and E.Cesari, Phys. Rev. B55,11068 (1997).

[11] A.Zheludev, S.M.Shapiro, P.Wochner, A.Schwartz, M.Wall and L.E.Tanner, Phys. Rev. B51, 11310 (1995).

[12] A.Zheludev, S.M.Shapiro, P.Wochner and L.E.Tanner, Phys. Rev. B54, 15045 (1996).

[13] E.Obradó, A.Gonzàlez-Comas, Ll.Mañosa and A.Planes, J. Appl. Phys. 83, 7300 (1998).

[14] U.Stuhr, P.Vorderwisch, V.V.Kokorin and P.A. Lindgård, Phys. Rev. B 56, 14360 (1997).

[15] F.Zuo, X.Su and K.H.Wu, Phys. Rev. B 58 (1998) 11127.

[16] A.Gonzàlez-Comas, E.Obradó, Ll.Mañosa, A.Planes, V.A.Chernenko, B.Hattink and A.Labarta, Phys. Rev. B (submitted).

[17] P.J.Webster, K.R.A.Ziebeck, S.L.Town and M.S.Peak, Phil. Mag. B49, 295 (1984).

[18] V.A.L'vov, E.V.Gomonaj and V.A.Chernenko, J.Phys.: Condens. Matter 10 (1998) 4587. 